\title{
Abstract \\ New developments in SKED and in minicomputer systems
}

RONALD M. KADDEN, Veterans Administration, Montrose, New York 10548

The discussion included projections into the future and problems currently being encountered in such areas as interfacing, peripheral devices, alternative computer systems, and user-produced F3 subroutines. The existence of a SKED Users Group was publicized as a means of communicating new developments and problem solutions throughout the year. 